\title{
CAMBIOS AMBIENTALES EN EL CHACO ARGENTINO Y BOLIVIANO EN LOS ULTIMOS MILES DE AÑOS
}

\section{Martín Iriondo}

\section{Introducción}

El Chaco es una extensa planicie interior de Sudamérica formada por los megaabanicos aluviales, los de los ríos Salado, Bermejo, Pilcomayo y Parapetí. Los ríos Dulce (en el suroeste) y Grande (en el noroeste) forman áreas de transición con la Planicie Amazónica y con el Sistema de Sierras y Bolsones del oeste argentino respectivamente.

El Chaco es una llanura de $840.000 \mathrm{~km}^{2}$ ubicada alrededor del trópico sudamericano, que se extiende por tres países. Desde el punto de vista climático constituye una excepción, pues en el contexto de la circulación general de la atmósfera se encuentra ubicado en la faja de los grandes desiertos tropicales. Sin embargo, no parece haber sido un desierto propiamente dicho en el pasado, por lo menos a lo largo de los últimos cientos de miles de años.

Esta región ha sufrido cambios climáticos comprobados en los últimos miles de años, que han oscilado entre tropical húmedo y subtropical semiárido en general, con un gradiente de humedad este-oeste. En esta contribución se describen los episodios climáticos más importantes desde el punto de vista de la ocupación humana del territorio. Dichas reconstrucciones climáticas resultaron de investigaciones geomorfológicas y sedimentológicas desarrolladas por este autor y colaboradores a lo largo de los últimos veinte años. También se han incorporado resultados de colegas de otras disciplinas, con la finalidad de construir escenarios coherentes para cada caso.

No se ha incorporado en este artículo la fundamentación técnica de cada afirmación, ya que las validaciones s encuentran en la bibliografía citada y resultaría engorroso repetirla acá. Para profundizar en el tema se puede recurrir a dos artículos titulados “Geomorphology and late Quaternary of the Chaco (Iriondo, 1993) and "Quaternary climates of South America (Iriondo, 1999).

\section{Clima actual y vegetación}

El clima del Chaco es tropical semiárido a tropical húmedo. Está caracterizado por un intercambio permanente de masas de aire tropical y austral. Durante períodos de viento norte, la temperatura puede subir a $40^{\circ} \mathrm{C}$ o más en una hora, incluso durante los meses frescos de agosto y septiembre. Las lluvias son generalmente intensas y pertenecen a dos tipos de precipitación: frontal, producida por el avance de masas de aire frío del sur o caliente del norte; y de convección, originadas en procesos convectivos locales dentro de una masa de aire tropical húmedo estacionada sobre la región, particularmente en verano.

En este último caso pueden caer más de 200 milímetros en unas pocas horas en áreas muy limitadas, mientras que a pocos kilómetros de distancia brilla el sol. En el Chaco 
bolviano la temporada de lluvias comienza en octubre y termina en marzo y los inviernos son secos, la estacionalidad es muy marcada. En el sector oriental argentino dicha diferencia estacional es bastante menos marcada.

Las temperaturas medias varían entre $24^{\circ} \mathrm{C} \mathrm{y} 30^{\circ} \mathrm{C}$ en verano (según las localidades) con máximas por sobre los 40 grados todos los años. Las amplitudes diurnas son grandes en el oeste, alcanzando los 15 y 20 grados, con importante enfriamiento nocturno provocado por radiación, que produce heladas en algunos inviernos. Las condiciones son más suaves en el este, debido a la mayor humedad del aire.

La vegetación está caracterizada por bosques, sabanas y asociaciones de pantanos. En el Chaco oriental los bosques presentan alta diversidad específica y frecuentemente los grandes pantanos son cubiertos por vegetación flotante y plantas palustres. Como consecuencia del gradiente climático, en el oeste semiárido la vegetación está representada por solamente diez especies arbóreas xerofíticas, cactus y pajonales (Cabrera y Willnick, 1930). A nivel local, la vegetación depende simplemente del tipo y altura relativa del substrato: bosque complejo en albardones, palmeras en las pendientes intermedias, pajonal en las depresiones, etc. De manera que, como el substrato sedimentario ha sido formado en las sucesivas fases climáticas y ambientales ocurridas en los últimos miles de años, la geografía física actual es el resultado directo de aquellas.

El Chaco es una provincia biogeográfica homogénea y bien definida del Dominio Chaqueño, que a su vez es un importante nivel biogeográfico que cubre un amplio sector de Sudamérica. La Provincia Chaqueña se caracteriza por un escaso número de formas endémicas a nivel familia; son típicas las Leguminosiae, Mimosoidae y Edentata, Chiropterae, Tinamidae y un pez pulmonado (Lepidosiren paradoxa). Entre los insectos son abundantes las hormigas (Atta) y las termitas (Cornitermes) (Cabrera y Willnick, op.cit.).

En mi opinión, desde un punto de vista evolutivo, el Dominio chaqueño puede ser interpretado como un ambiente secundario, derivado de la interacción de los dos grandes polos neotropicales: Amazonia y Patagonia. Considerando que los sistemas geológicos y climáticos actuales de Sudamérica fueron establecidos aproximadamente en el Mioceno (y consecuentemente los Dominios Patagónico y Amazónico), se puede postular una edad pliocena (entre 5 millones de años y 2 millones de años) para la identidad biogeográfica del Chaco.

\section{El Chaco occidental}

El Chaco occidental se caracteriza por su clima semiárido; está limitado hacia el oeste por las Sierras Subandinas, donde ocurren precipitaciones del orden de los 1000 a 2500 milímetros anuales. Está dominado por la dinámica de los grandes ríos alóctonos que poseen fuerte tendencia a la divagación lateral. Dichos ríos llegan a la llanura desde sus cuencas montañosas a lo largo de profundos cañones, transportando arena cuarzosa fina, bien seleccionada. A pesar de la elevada capacidad de transporte de esas corrientes, las fracciones granulométricas gruesas tales como cantos rodados están ausentes en general. La única excepción es el río Bermejo. El levantamiento de las montañas se produjo durante 
el Plioceno, de manera que todo el escenario natural chaqueño parece haberse generado en esa época.

Los grandes ríos atraviesan la región hacia el este, a lo largo de fajas complejas caracterizadas por cauces abandonados y formas menores. Ocasionalmente se produce un proceso mayor de avulsión, durante el cual un río abandona un cauce y ocupa otra depresión (a veces lejana) en un proceso rápido que puede ser de pocos días o algunos años. El Bermejo abandonó, a mitad del siglo XIX, un trecho de más de $200 \mathrm{~km}$ de cauce, abriéndose camino por otra dirección (Iriondo, 1990). En la actualidad el Pilcomayo sufre un proceso similar a lo largo de algunos años (Iriondo, 1997)

Durante los climas más secos que el actual los ríos que descienden del oeste han sido más pequeños y más divagantes, hecho que se deduce de la presencia de numerosos paleocauces de reducidas dimensiones en toda la llanura del oeste. Esos paleocuaces tienen un patrón distributario a gran escala; son especialmente visibles en los abanicos de los ríos Pilcomayo y Salado. Cuando la sequedad del clima llegó a valores máximos se estableció en el Chaco occidental una dinámica eólica con movilización de polvo y arena, producida por vientos fuertes y secos del norte.

\section{El Chaco oriental}

El Chaco oriental está constituído por las regiones distales de los abanicos aluviales, formadas por áreas pantanosas atravesadas por antiguas fajas fluviales de los grandes ríos. El clima actual es húmedo, con exceso de precipitaciones, a lo que se le agregan los desbordes de los cauces principales producidos por agua llegada de las montañas del oeste. La infiltración del agua es prácticamente nula, debido a que el terreno superficial está compuesto por arcillas impermeables hasta una profundidad de 12 a 20 metros. La pendiente, extremadamente baja, no es adecuada para la evacuación de los excesos hídricos. En consecuencia, aparecen pantanos permanentes y temporarios, densamente cubiertos por la vegetación palustre y flotante.

En esos ambientes la evapotranspiración es varias veces mayor que la evaporación potencial, y la materia orgánica se acumula en el ambiente anaeróbico del fondo de los pantanos. Neiff (1986) distingue pantanos temporarios ("bañados") y permanentes ("esteros"). Los temporarios no tienen sedimentos propios, están alimentados por agua de lluvia y el agua permanece estancada hastas 6 meses en forma de lámina de hasta 30-40 $\mathrm{cm}$ de espesor, con períodos de baja recurrencia. Existe un corto período de deficiencia de oxígeno, producido por la degradación de la vegetación en el comienzo de la fase de anegamiento.

Los pantanos permanentes están mejor delimitados; tienen orillas mejor definidas y están poblados por vegetación adaptada para vivir en condiciones de anaerobiosis prolongada por su sistema radicular. También están adaptados a los incendios periódicos, un mecanismo natural en estos ambientes. Los pantanos más extensos, entre 100-200 km de longitud y 3-10 km de ancho, tienen generalmente una profundidad menor a 1 metro. Los pantanos cubren un área de $125.000 \mathrm{~km}^{2}$ en los abanicos de los ríos Bermejo y Pilcomayo. 
Dichos pantanos forman los tramos superiores de redes fluviales locales, desarrollados en los mega-abanicos durante el clima húmedo actual. Los colectores de esas redes locales son arroyos muy divagantes, de 2 a $5 \mathrm{~m}$ de profundidad y 20 a $60 \mathrm{~m}$ de ancho (Orfeo, 1986) que corren por antiguos cauces de los ríos alóctonos mayores bordeados por albardones. Constituyen un tipo especial de río subajustado, en el cual el cauce antiguo tuvo una cuenca mucho mayor que el cauce actual. En las inundaciones extraordinarias el agua cubre toda la región; los únicos elementos geomorfológicos emergentes son los antiguos albardones.

\section{Los abanicos aluviales}

Los grandes abanicos aluviales de los ríos chaqueños (o mega-abanicos) son sistemas complejos, formados por unidades sedimentarias y morfológicas de diferentes edades y contextos climáticos. Aunque son similares entre sí y sus características generales, cada uno de ellos presenta particularidades significativas. De sur a norte los abanicos son los siguientes: Salado, Bermejo, Pilcomayo y Parapetí. Los ríos Grande (al norte) y Dulce (al suroeste) ocupan franjas marginales.

El abanico del río Salado - Mide aproximadamente $650 \mathrm{~km}$ de longitud por $150 \mathrm{~km}$ de ancho en su parte distal. Está compuesto por un conjunto de subsistemas menores: abanicos, fajas y depósitos lacustres de diverso tipo. Sus depósitos sedimentarios están formados predominantemente por limos, arcilla illitica y arena fina. Entre las sales solubles los cloruros son absolutamente dominantes, seguidos en abundancia por los carbonatos. Los abanicos menores se han formado durante períodos secos, en lugares donde los movimientos neotectónicos formaron depresiones de algunos miles de kilómetros cuadrados. En su región distal, en la provincia de Santa Fe, el abanico del Salado presenta un sector compuesto por un conjunto de paleocauces formados en el Pleistoceno superior, durante una época suficientemente húmeda como para permitir la conservación de cauces permanentes (el Pleistoceno abarca desde unos dos millones de años atrás hasta diez mil años antes del presente). Aunque están enterrados por una carpeta de loess, son visibles en superficie; tienen trazado irregular y poco divagante. En general, su ancho no sobrepasa los 200 metros. Los cauces de distinta edad y dirección se presentan parcialmente entrecruzados y superpuestos, en una trama irregular. Algunos de ellos son portadores de agua dulce, lo que les confiere un gran valor potencial en una zona con graves problemas de agua potable.

Son interesantes en la región distal las llamadas "isletas", pequeñas extensiones de 40 a 80 metros de diámetro y 30 a $50 \mathrm{~cm}$ de altura, cubiertas por espeso bosque, denominadas "isletas". Las isletas se originan por la actividad biológica de las hormigas del género Atta; una sola colonia de ellas puede formar un túmulo de varios metros de diámetro (Bonetto, 1959). El volumen de tierra removida y alterada por cada hormiguero es de varios metros cúbicos, formándose un sedimento blando y húmedo, compuesto por grumos redondeados menores a $2 \mathrm{~mm}$ y alta porosidad. Este material forma un substrato adecuado para árboles y arbustos, y sería probablemente adecuado para asentamientos humanos en sociedades primitivas. 
El abanico del río Bermejo - El abanico del Bermejo tiene su ápice cerca de Embarcación, en Bolivia, y se extiende hacia el este y sudeste hasta la faja Paraguay-Paraná, a 650 kilómetros de distancia. Un gran número de cauces abandonados ("ríos muertos") holocenos puede ser observado en su superficie. El rumbo general de los mismos es noroeste-sudeste y pueden ser trazados a lo largo de decenas de kilómetros; esa morfología es característica de gran parte del Chaco Occidental y Central. En el sur se ha desarrollado un gran lóbulo de derrame que llega hasta la provincia de Santa Fe. En la zona de Charadai, en la parte oriental del lóbulo, se ha implantado un ambiente de pantanos probablemente a fines del Pleistoceno, que ha permanecido hasta la actualidad. En dicho ambiente se depositaron de 6 a 12 metros de espesor de arcillas limosas gris verdosas y marrón rojizas endurecidas, en un bloque hundido de $5.000 \mathrm{~km}$ de extensión. Varias cuencas fluviales locales se han desarrollado en la región oriental del abanico del Bermejo (Tapenagá, El Rey, Los Amores, Negro) con rasgos semejantes entre sí.

A la altura de Las Lomitas el río Bermejo tiene un cauce de $1 \mathrm{~km}$ de ancho, muy divagante, con alta carga de sedimentos en suspensión y arena cuarzosa muy fina transportada por arrastre. Forma bancos planos de cientos de metros de largo, incluyendo en la arena numerosos troncos y ramas de palo bobo (Tessaria integrifolia). La arena se agrieta profundamente al secarse, formando polígonos de hasta un metro de diámetro. Es probable que los mismos sean originados por el alto porcentaje de coloides que transporta el río.

El abanico del río Pilcomayo - El Pilcomayo ha formado el abanico aluvial más importante del Chaco y uno de los mayores abanicos de Sudamérica. Se trata de uno de los pocos o el único mega-abanico activo de este continente. Su superficie total es de $210.000 \mathrm{~km}^{2}$. Tiene una amplia cuenca montañosa en la Cordillera Oriental y en las Sierras subandinas bolivianas. Atraviesa esas montañas en grandes meandros incididos, en una situación claramente antecedente, es decir que el río es más antiguo que las montañas y fue excavando su cauce a medida que estas se elevaban. Sus afluentes subandinos, por el contrario, son subsecuentes o sea más jóvenes que las sierras.

Los sedimentos del río en Villa Montes estan compuestos por arena muy fina cuarzosa. El caudal máximo es 45 veces mayor que el mínimo. En la parte alta del abanico el Pilcomayo ha mudado de cauce varias veces durante el clima húmedo actual. Durante los últimos siglos el río Pilcomayo desembocó en una depresión de origen tectónico, de unos $15.000 \mathrm{~km}^{2}$ de superficie, denominada "estero Patiño", depositando en ella grandes cantidades de arena muy fina y limo. El mecanismo de colmatación de ese estero fue estudiado por Cridini (1974). Hacia 1980 el estero Patiño terminó de rellenarse completamente. En consecuencia, el Pilcomayo comenzó a rellenar su cauce aguas arriba, desbordando el agua a la planicie que lo rodea. Dicho mecanismo provocó el retroceso del punto de desborde hacia aguas arriba en sucesivas crecientes, entre 10 y $35 \mathrm{~km} / \mathrm{a} n$ o. Finalmente, el fenómeno alcanzó el punto tripartito de la frontera (Argentina-BoliviaParaguay) y se produjo una avulsión hacia el norte y el sur. En un esquema simplificado, el desarrollo del mega-abanico del Pilcomayo ha seguido una secuencia de dos mecanismos 
contrastados: a) desarrollo de fajas fluviales estables durante períodos húmedos, como el actual; b) sedimentación generalizada mediante una sucesión de cauces efímeros en los períodos climáticos secos. En ambos casos, desarrollo de pantanos de distintos tipos en los bloques hundidos.

El abanico del río Parapetí - El Parapetí forma un abanico aluvial de varias decenas de miles de kilómetros cuadrados en Bolivia y Paraguay. Pertenece en parte a la cuenca del Paraná y en parte a la cuenca amazónica. Actualmente, su cauce permanente desemboca en el bañado del Izozog, que deriva en el río Mamoré. En la época de creciente aparece un derrame importante que forma el río Timane, que se dirige hacia el este hacia el río Paraguay. De acuerdo a Huamán et al. (1975) se pueden reconocer cuatro unidades morfológico/ sedimentarias en la superficie del abanico: a) depósitos aluviales antiguos; b) llanura eólica; c) faja aluvial actual del río; y d) bañado del Izozog.

La llanura eólica cubre un área de $25.000 \mathrm{~km}^{2}$ en el este de Bolivia y el noroeste de Paraguay. Está formada por arena fina en grandes dunas parabólicas de orientación norte-sur, de varios kilómetros de longitud individual. Dichas dunas corresponden a la movilización eólica generalizada ocurrida en el Holoceno superior entre 3400 años antes del presente y 1400 años antes del presente (Servant et al., 1981). Nótese que se trataba de vientos fuertes y secos provenientes de la planicie amazónica

\section{La faja Paraguay - Paraná}

El límite oriental del Chaco está marcado por los ríos Paraguay y Paraná, desde la frontera paraguayo-boliviana hasta la ciudad de Santa Fe. Durante el Cuaternario, estos ríos han formado una faja de sedimentos y geoformas de orientación norte-sur con características bien definidas, diferentes al resto del Chaco. Está compuesta por estratos gruesos de arena cuarzosa y mezcla de minerales arcillosos; el relieve local es moderado a bajo, en contraposición con la marcada horizontalidad de los mega-abanicos del resto del Chaco. Esta faja es estrecha (5 a $10 \mathrm{~km}$ de ancho) en el norte y se amplía considerablemente en la provincia de Santa Fe, alcanzando un ancho de 100 kilómetros.

\section{Los climas cuaternarios}

El Período Cuaternario se ha caracterizado en la historia geológica de la Tierra por fuertes cambios climáticos; los ejemplos más importantes de los mismos han sido las glaciaciones de Eurasia y América del Norte. En América del Sur ocurrieron cambios importantes, aunque no se caracterizaron por grandes cambios de temperatura sino por oscilaciones de primer orden en las precipitaciones. Así, en Europa el Cuaternario se define por una sucesión de épocas glaciales e interglaciales; en nuestro continente por una secuencia de climas húmedos y secos en cada región.

Si se visualiza al clima de todo el continente o de una porción importante del mismo, pueden definirse en la actualidad "provincias climáticas", regiones en las que el clima es 
relativamente homogéneo y diferente al de los territorios vecinos. Por ejemplo "Pampa Húmeda" o "Patagonia Extraandina" (Iriondo y García, 1993). En ese contexto regional, cuando se produce un cambio climático, una provincia determinada crece a expensas de las provincias vecinas o viceversa. En los períodos secos (correspondientes a las glaciaciones en otros continentes y en el sur sudamericano) la provincia climática patagónica avanzó hacia el noreste cientos de kilómetros. En los péríodos húmedos (interglaciales desde el punto de vista global) el clima actual de Misiones se extendió hasta el sudoeste de Corrientes y mitad oriental de Entre Ríos.

\section{Secuencia climática y ambiental}

Existe actualmente un razonable conocimiento de la sucesión de climas ocurridos en la llanura argentina en los últimos cien mil años (Iriondo y Kröhling, 1995). En lo que respecta al Chaco, se dispone de algunas evidencias directas y además se puede correlacionar con los resultados obtenidos en la región pampeana y en la Mesopotamia. El período de tiempo que ofrece mayor interés en nuestro caso es el que se extiende desde el Ultimo Máximo Glacial hasta el presente.

El Ultimo Máximo Glacial - Fue un enfriamiento general de la atmósfera que se extendió en nuestro país entre los 36.000 y los 8500 años antes del Presente (a.A.P.). Se formaron campos de hielo en la Patagonia y crecieron glaciares de valle a lo largo de los Andes y otras montañas altas. Las líneas climáticas migraron hacia el noreste unos 1.000 kilómetros, llegando el límite de la Patagonia hasta la actual ciudad de Santa Fe. Las precipitaciones anuales en esa zona llegaban a los 200-300 milímetros (actualmente llueve más de 1.000 $\mathrm{mm} / \mathrm{a}$ ). La estructura meteorológica dominante era el viento Pampero, potenciado al cruzar las masas de aire del Pacífico por sobre el hielo patagónico. De manera que se trataba de un clima definidamente seco. Por otro lado, la temperatura media anual fue probablemente solo 2,5 a $3^{\circ} \mathrm{C}$ inferior a la actual (Iriondo y García, 1993). Se puede deducir de lo anterior para el Chaco Oriental un escenario geográfico similar al que actualmente existe en la provincia de La Pampa.

El Chaco Occidental tuvo una dinámica diferente durante el UMG. El clima era semidesértico a desértico y vientos secos y fuertes del norte (curiosamente originados en la planicie amazónica) formaron campos de dunas de gran tamaño en la llanura boliviana y una orla de loess en los valles adyacentes y en el frente del sistema ubicado en Salta-Tucumán (Iriondo, SAES). Campos de arena menores fueron formados también en el sector argentino hasta la longitud de Las Lomitas. El río Bermejo en Salta desarrolló un sistema de cauces efímeros, pequeños, con capacidad de transportar solamente el $18 \%$ del caudal medio actual. Puede presumirse que los "surazos", masas de aire polar que alcanzan raramente la región y provocan heladas en el invierno, fueron frecuentes durante este período. 
El Hypsithermal " "Optimum Climaticum" - Hacia el 8.500 A.P. se produjo un calentamiento general de la atmósfera que fue acompañado por mayores precipitaciones en el Chaco y en la Pampa, que se extendió hasta el 3.500 A.P. Su comienzo produjo la extinción de los últimos restos de la megafauna pleistocena y dentro de él ocurrió el poblamiento humano generalizado de la región pampeana y áreas vecinas. Las precipitaciones en el Chaco salteño y boliviano aumentaron hasta el triple de los valores actuales, generándose un suelo que requiere más de $800 \mathrm{~mm} / \mathrm{a}$ durante dos o tres milenios para desarrollarse. Las temperaturas fueron unos 2 grados más altas que las actuales (1,8 en Cipolletti) y el complejo de esteros y pantanos del Chaco Oriental probablemente se extendió hacia el oeste. Desde un punto de vista ambiental, es razonable suponer que el clima actual de la ciudad de Formosa se desplazó hasta la ciudad de Santiago del Estero y aun más al sur, y que el Chaco argentino tuvo un clima similar al que hoy se registra en el Paraguay Oriental, con muy escasa influencia de las masas de aire del sur. Los Yungas, que actualmente llegan hasta Tucumán, alcanzaron las sierras de Córdoba. Suelos rojos típicos de Misiones y el Brasil se formaron hasta la zona de Esquina, en el extremo suroeste de Corrientes La fauna brasileña y paraguaya ocupó todo el Chaco.

El clima seco del Holoceno superior - Entre los años 3500 y 1400 A.P. (o sea hasta el año 600 de nuestra era) se implantó un clima seco, básicamente semiárido, sobre la llanura chaco-pampeana (Iriondo, 1990). Este pulso climático particular fue provocado por la aparición de un anticiclón estacional que bloqueaba la circulación general de la atmósfera, impidiendo las precipitaciones frontales. Este fenómeno ha aparecido esporádicamente en los años muy secos del siglo XX. La circulación anticiclónica de los vientos tiene sentido anti-horario en el Hemisferio Sur. En este caso se produjo la erosión generalizada del horizonte superficial del suelo y la acumulación de una capa de polvo de 20 a $40 \mathrm{~cm}$ de espesor en toda la región. Se formaron campos de dunas parabólicas (indicadoras de semiaridez) en varias areas, siendo algunos de los más importantes los del río Parapetí. La localidad de Las Lomitas debe su nombre a un pequeño campo de dunas de esta edad. Se formaron acumulaciones sedimentarias locales de dos o más metros de altura en la orilla de las lagunas del Chaco santafesino, denominadas "lunetas" o "dunas de arcilla", en las cuales se encuentran generalmente restos arqueológicos. Una reconstrucción climática de ese período sugiere un clima semiárido relativamente homogéneo para toda la región chaco-pampeana, con precipitaciones de 300-400 mm/a, mayor amplitud térmica que en la actualidad, capas freáticas profundas, ausencia de bosques en el Chaco y ausencia de pantanos en Corrientes y el Paraguay.

De acuerdo datos faunísticos holocenos y a la analogía meteorológica de los años secos del siglo XX, la temperatura fue similar o algo mayor que la actual, y debe visualizarse a esa época como un avance climático del Chaco Occidental hacia el Oriental. La acción dominante del viento en el modelado del paisaje, que requiere suelo desnudo, indica que el bosque chaqueño desapareció por lo menos en gran parte, así como también el sistema de esteros. Probablemente los grandes ríos Bermejo y Pilcomayo constituyeron importantes corredores y refugios del ecosistema y de la población humana vinculada al mismo. 
El Máximo Medieval - Existen varios indicadores climáticos que marcan un calentamiento acompañado por aumento de humedad para el período 1400-800 a.A.P. , o sea entre los años 600 y 1400 de nuestra era. Dichos indicadores son pedológicos, faunísticos, geológicos y arqueológicos. En el sur de San Luis se formaron pequeñas lagunas permanentes en lugares hoy desérticos y la selva misionera alcanzó la latitud de Concordia, en Entre Ríos. Se produjo una migración generalizada de arcilla en los suelos (pedogénesis).

Esta fase climática fue una repetición del Hypsithermal con características menos fuertes. El clima tropical se instaló sobre todo el Chaco y las lluvias se hicieron más abundantes que las del siglo XX. Seguramente los esteros tuvieron una fase de agua abundante y probablemente la vegetación del este colonizó el oeste, aunque el período puede haber sido demasiado corto para ciertas especies de árboles. Es necesario notar que el fin de este período corresponde aproximadamente con la llegada de los conquistadores europeos a la región; y todo cambio climático supone per se fuertes tensiones y crisis ecológicas y ambientales.

La Pequeña Edad del Hielo - La Pequeña Edad del Hielo (PEH) es un período de enfriamiento y sequía que coincidió aproximadamente con el período histórico colonial de América. En la Argentina produjo avances glaciales en la Cordillera y aridez en las tierras bajas. Ha sido detectado desde tiempo atrás por varios investigadores (ver Iriondo y Krohling, op.cit.). El clima patagónico avanzó hasta el límite entre las provincias de Santa Fe y Buenos Aires. El escenario general indica una dinámica similar a la del Ultimo Máximo Glacial, aunque atenuada. Depósitos de arena eólica en esa zona indican vientos dominantes del suroeste, o sea el Pampero. Las sequías eran tan severas que el río Salado de Buenos Aires llegaba a secarse. Se estima que la temperatura media fue $1^{\circ} \mathrm{C}$ más baja que la del siglo XX.

Existen interesantes crónicas de la época, como la del Padre Parras (1943), que establecen que la laguna Mar Chiquita de Córdoba no existía como cuerpo de agua, sino que era un gran barreal rodeado de arenales, cruzado por el camino Santa Fe-Santiago del Estero. El paisaje del norte de Buenos Aires y sur de Santa Fe era "pelado y desapacible" hasta el río Carcarañá, cuya agua era demasiado salada para que bebieran los caballos. Actualmente esa zona es una pradera con lluvias de 800 a $1000 \mathrm{~mm}$ anuales. La travesía entre Córdoba y Santa $\mathrm{Fe}$ era muy difícil para los jinetes por falta de agua, por lo que generalmente se esperaba que cayera una lluvia fuerte antes de emprender el viaje. Según Parra, que vivió a mediados del siglo XVIII "el viento n Buenos Aires jamás cesa, ni siquiera por dos horas".

Azara y Darwin también mencionan grandes sequías; el primero de ellos marca en las Misiones el límite de la selva al norte de la línea actual. El caudal del río Paraná era tan modesto que en algunos años dejaba de ser navegable, excepto en "botecillos" (hoy en día es navegado por barcos de ultramar hasta Asunción). Esto indica que el clima seco de la PEH afectó toda la cuenca del Paraná, incluyendo sur de Brasil y Paraguay. De manera que el ambiente general en el Chaco fue de semiaridez y predominio de vientos del sur. La 
PEH finalizó hacia el año 1800 de nuestra era, es decir unos 200 a.A.P. y fue reemplazado por el clima característico del siglo XX.

\section{Referencias bibliográficas}

Cabrera, A. y Willinck, A. 1980. Biogeografía de América Latina. Monografía 13, Serie Biología, OEA, 122pp., Washington.

Huamán, A., Ballivián, G., Méndez, O. Y Algañaraz, J. 1975. Levantamiento integrado de los recursos naturales del sector occidental de la Provincia de Cordillera, parte llana. Corporación de Desarrollo Regional de Santa Cruz, 30 pp., Santa Cruz.

Iriondo, M. 1990. "A late Holocene dry period in the Argentine plains". Quaternary of South America and Antarctic Península, 7:197-218, A.A.Balkema, Rotterdam.

Iriondo, M. 1993. "Geomorphology and late Quaternary of the Chaco (South America)". Geomorphology, 7:289-303. Elsevier Sci. Publ., Amsterdam.

Iriondo, M. 1995. "El Cuaternario del Chaco". En: Argollo y Mourguiart (Eds.): Climas Cuaternarios en América del Sur ORSTOM/Universidad Mayor de San Andrés, 263-282, La Paz.

Iriondo, M. 1997. "Models of deposition of loess and loessoids in the upper Quaternary of South america". Journal of South American Earth Sciences, 10:71-79, Elsevier Sci. Publ., Amsterdam.

Iriondo, M. 1999. "Climatic changes in the South American plains: record of a continent-scale oscillation”. Quaternary International, 57/58:93-112. Pergamon Press, Oxford.

Iriondo, M. y García, N. 1993. "Climatic variations in the Argentine plains during the last 18,000 years". Palaeogeography, Palaeoclimatology, Palaeoecology, 101:209-220, Elsevier Sci. Publ., Amsterdam.

Iriondo, M. y Kröhling, D., 1995. "El Sistema Eólico Pampeano”. Com. Mus. Prov. Ciencias Naturales Florentino Ameghino, 5(1):1-68, Santa Fe.

Neiff, J. 1986. "Sinopsis ecológica y estado actual del Chaco oriental”. Ambiente Subtropical, 1:536, Corrientes.

Orfeo, O. 1986. "Evaluación de sólidos suspendidos en algunos ríos del noreste argentino". Rev. Asoc. Arg. de Mineralogía, Petrología y Sedimentología, 17:61-66, Buenos aires.

Parras, P. 1943. "Diario y derrotero de sus viajes". Editorial Solar, 251 pp., Buenos Aires.

Servant, M., Fontes,J., Rieu, M. y Salliege, J. 1981. "Les dines eoliennes du Soudoues de l'Amazonie". Comptes Rendues Acad. Sci. Paris, 292(II):1295-1297, París.

\section{Resumen}

El Chaco es una gran llanura tropical ubicada en el interior de Sudamérica y que cubre partes de Argentina, Bolivia y Paraguay. Tiene $840.000 \mathrm{~km} 2$ de extensión y está caracterizado por bosques, sabanas y grandes pantanos, lo que le otorga una marcada identidad climática y biogeográfica. Está compuesto por cuatro mega-abanicos formados por cuatro grandes ríos: Salado, Bermejo, Pilcomayo y Parapetí; los ríos Dulce y Grande recorren ambientes transicionales en el noroeste y suroeste. En el este se extiende una faja de territorio asociada a los ríos Paraguay y Paraná. Se han sucedido diferentes climas en el Chaco durante el Cuaternario superior. Los climas húmedos, como 
el actual, favorecieron el desarrollo de suelos y fajas fluviales estables; durante los climas secos a semiáridos aparecieron cauces efímeros y derrames de sedimentos sobre la llanura. Durante esos intervalos se formaron campos de dunas y mantos de loess en el oeste.

$$
<\text { Chaco }><\text { Cuaternario }><\text { Geomorfología }><\text { Cambios climáticos }><\text { Holoceno }>
$$

\begin{abstract}
The Chaco is a large tropical plain located in the interior of South America, consisting of parts of Argentina, Paraguay and Bolivia. It is $840,000 \mathrm{~km} 2$ in area and is characterized by forests, savannas and extensive swamps, which give it a marked climatic and biogeographic identity. It is formed by four mega-fans built by the major rivers in the region: Salado, Bermejo, Pilcomayo and Parapetí. The Grande and Dulce rivers occupy belts which are transitional with neighboring regions to the northwest and southwest. Humid climates, such as the present one, favoured the generation of soils and stable fluvial belts; drier climates led to widespread sedimentation along small ephemeral channels and large spill-outs. During such intervals, dune fields and loess mantles were formed in the western areas.
\end{abstract}

$<$ Chaco $><$ Quaternary $><$ Geomorphology $><$ Climatic changes $><$ Holocene $>$ 